\title{
The homeoprotein Dlx5 drives murine T-cell lymphomagenesis by directly transactivating Notch and upregulating Akt signaling
}

\author{
Yinfei Tan ${ }^{1, *}$, Eleonora Sementino ${ }^{1, *}$, Jinfei $\mathrm{Xu}^{1}{ }^{1}$, Jianming Pei $^{1}$, Zemin Liu ${ }^{1}$, Timothy \\ K. Ito ${ }^{1}$, Kathy Q. Cai ${ }^{2}$, Suraj Peri' ${ }^{3}$, Andres J.P. Klein-Szanto ${ }^{1,2}$, David L. Wiest ${ }^{4}$, \\ Joseph R. Testa ${ }^{1}$ \\ ${ }^{1}$ Cancer Biology Program, Fox Chase Cancer Center, Philadelphia, PA 19111, USA \\ ${ }^{2}$ Histopathology Facility, Fox Chase Cancer Center, Philadelphia, PA 19111, USA \\ ${ }^{3}$ Department of Biostatistics and Bioinformatics, Fox Chase Cancer Center, Philadelphia, PA 19111, USA \\ ${ }^{4}$ Blood Cell Development and Function Program, Fox Chase Cancer Center, Philadelphia, PA 19111, USA \\ *These authors contributed equally to this work
}

Correspondence to: Joseph R. Testa, email: Joseph.Testa@fccc.edu

Keywords: $T$ cell lymphoma, Notch, Akt, DIx5, homeobox

Received: November 09, $2016 \quad$ Accepted: January 11, $2017 \quad$ Published: January 21, 2017

\section{ABSTRACT}

Homeobox genes play a critical role in embryonic development, but they have also been implicated in cancer through mechanisms that are largely unknown. While not expressed during normal T-cell development, homeobox transcription factor genes can be reactivated via recurrent chromosomal rearrangements in human T-cell acute leukemia/lymphoma (T-ALL), a malignancy often associated with activated Notch and Akt signaling. To address how epigenetic reprogramming via an activated homeobox gene might contribute to T-lymphomagenesis, we investigated a transgenic mouse model with thymocyte-specific overexpression of the DIx5 homeobox gene. We demonstrate for the first time that DIx5 induces T-cell lymphomas with high penetrance. Integrated ChIP-seq and mRNA microarray analyses identified Notch1/3 and Irs2 as direct transcriptional targets of DIx5, a gene signature unique to Iymphomas from Lck-DIX5 mice as compared to T-cell lymphomas from LckMyrAkt2 mice, which were previously reported by our group. Moreover, promoter/ enhancer studies confirmed that DIx5 directly transactivates Notch expression. Notch1/3 expression and Irs2-induced Akt signaling were upregulated throughout early stages of $\mathrm{T}$-cell development, which promoted cell survival during $\beta$-selection of $\mathrm{T}$ lymphocytes. DIx5 was required for tumor maintenance via its activation of Notch and Akt, as tumor cells were highly sensitive to Notch and Akt inhibitors. Together, these findings provide unbiased genetic and mechanistic evidence that DIX5 acts as an oncogene when aberrantly expressed in $\mathrm{T}$ cells, and that it is a novel discovery that Notch is a direct target of DIx5. These experimental findings provide mechanistic insights about how reactivation of the DIx5 gene can drive T-ALL by aberrant epigenetic reprogramming of the T-cell genome.

\section{INTRODUCTION}

Aberrant expression of multiple NK-like (NKL) homeobox transcription factor genes is especially common in human T-cell acute leukemia/lymphoma (T-ALL), a malignancy often associated with activated Notch and Akt signaling [1]. While not expressed during normal T-cell development, NKL genes such as TLX1 (HOX11),
TLX2 (HOX11L1), and TLX3 (HOX11L2) may become transcriptionally activated in T-ALL [1]. Many of these developmentally related genes have also been implicated oncogenically, due to their reactivation via recurrent clonal chromosomal translocations and inversions. Typically, these rearrangements juxtapose $\mathrm{T}$ cell receptor regulatory sequences adjacent to homeobox loci such as HOX11 [2] and HOXA10 [3] leading to their upregulation. To date, 
however, little is known about oncogenic mechanisms and direct targets of these homeobox transcription factors in T-ALL.

The DLX family of homeodomain proteins also belong to the NKL superfamily. DLX homeoproteins play a role in bone formation, neurogenesis and hematopoiesis [4]. DLX5 was first identified as the mediator of bone morphogenetic protein (BMP) signaling and shown to regulate osteoblast differentiation, and Dlx5 knockout mice exhibited defects in facial-cranial development [5]. Recently, DLX family members have been implicated in oncogenesis. For example, DLX5 is abundantly expressed in a subset of adult human T-cell lymphomas [6], and DLX5 may contribute to tumorigenesis by directly regulating MYC expression [7]. The role of DLX homeoproteins has also been extended to other malignancies. In lung cancer, upregulated expression of DLX5 is predictive of a poor prognosis, and knockdown of $D L X 5$ suppresses lung tumor cell proliferation [8]. In breast cancer, $D L X$ homeoproteins have been shown to enhance metastatic potential, and DLX4 is capable of regulating epithelial-to-mesenchymal transition by augmenting TWIST levels [9]. Similarly, in glioblastoma patients, upregulation of DLX2 promotes tumor cell proliferation and is associated with reduced patient survival [10]. In ovarian cancer, DLX5 promotes cell proliferation via upregulation of AKT signaling through the direct transactivation of insulin receptor substrate 2 (IRS2) [11].

In mice, Dlx5 is normally expressed in adult bone marrow and fetal liver hematopoietic cells, but its expression is absent in Thy1-positive thymocytes [12]. Lck-MyrAkt 2 transgenic mice expressing a constitutively active (myristylated) form of the Akt2 kinase specifically in immature $\mathrm{T}$ cells develop a high incidence of thymic T-cell lymphomas. These tumors frequently harbor a somatic, clonal inversion of chromosome 6 that results in the juxtaposition of enhancer elements in the T-cell receptor (TCR) $\beta$-chain gene, Trb, and Dlx5 [6]. This rearrangement in Lck-MyrAkt2 mice results in high levels of expression of Dlx 5 in a tissue where it is not normally expressed. This reactivation of Dlx 5 was proposed to facilitate tumor development by interfering with T-cell differentiation and providing a second "hit" critical in the malignant transformation of thymocytes.

To address whether Dlx5 itself could represent a direct driving force in T-ALL, and how epigenetic reprogramming via a homeobox gene might contribute to T-lymphomagenesis generally, we generated a transgenic mouse model with thymocyte-specific overexpression of $D l x 5$, and we found that these Lck-Dlx 5 mice develop thymic lymphomas with high penetrance. The tumors that arise have constitutive activation of Akt in association with loss of Pten, and are highly sensitive to combinatory inhibition of Myc and Akt signaling [13]. We now report that Notch1/3 expression and Akt signaling are activated throughout $\mathrm{T}$ cell development in $L c k-D l x 5$ mice, and that tumor formation is associated with further intensification of Notch and Akt signaling. While Notch1 is regarded as the master oncogene in T-ALL [14], an in vivo mechanism responsible for its aberrant upregulation has not been previously reported. Using an unbiased, integrated genomic approach, we demonstrate for the first time that Notch1, Notch3, and Irs 2 are direct transcriptional targets of Dlx 5 in thymic T cells. Collectively, the experimental findings presented here provide mechanistic insights about how the reactivation of $D l x 5$ gene can drive T-ALL through aberrant epigenetic reprogramming.

\section{RESULTS}

\section{Lck-Dlx 5 transgenic mice develop disseminated T-cell lymphomas}

Lck-Dlx5 transgenic mice were generated by injecting the Lck-Myc-Tag-Dlx5 DNA fragment into blastocysts. Flow cytometric analysis revealed that non-malignant thymic $\mathrm{T}$ cells from all developmental stages expressed Myc-Tag Dlx5 protein (Figure 1A; Supplementary Figure 1A). Lck-Dlx 5 mice from each of four founders developed thymic lymphomas with high penetrance, and all tumors retained expression of Myctag Dlx5 (Figure 1B). Median survival of Lck-Dlx5 mice founder line F86 was 41 weeks, F63 was 37 weeks and F84 was 32 weeks (Figure 1C, Supplementary Figure 1B). Primary tumors were diagnosed as thymic T-cell lymphomas based on H\&E staining. The tumors often showed dissemination to the lung, with spreading to liver, kidney, spleen, marrow, and other organs in some animals (Figure 1D). Invasive tumor cells were mostly localized around blood vessels in lung, liver and kidney. IHC confirmed that primary and disseminated tumor cells were of T-cell origin, based on positive staining for CD3 (Supplementary Figure 1C), and that malignant cells were immature T cells, as they were CD4+CD8+ double positive (DP), similar to lymphoma cells in Lck-MyrAkt2 mice (Supplementary Figure 1D).

\section{Lymphomas in Lck-Dlx 5 mice exhibit a unique signature characterized by alterations of Notch and Akt}

Karyotypic analysis revealed that trisomy 15 was the only recurring abnormality in Lck-Dlx 5 lymphomas (Supplementary Table 1; Supplementary Figure 2A). Array-CGH analysis uncovered unique rearrangements of Tcrb and Tcra loci in each Lck-Dlx 5 lymphoma examined, indicative of monoclonal proliferation of a malignant $\mathrm{T}$ cell in each tumor (Supplementary Figure 2B). We used Affymetrix microarrays to assess mRNA expression patterns in Lck-Dlx 5 lymphomas. These studies revealed recurrent upregulation of Notch1, Notch3, Hes-1, and Ccnd1 in tumors from Lck-Dlx 5 mice, as compared to 

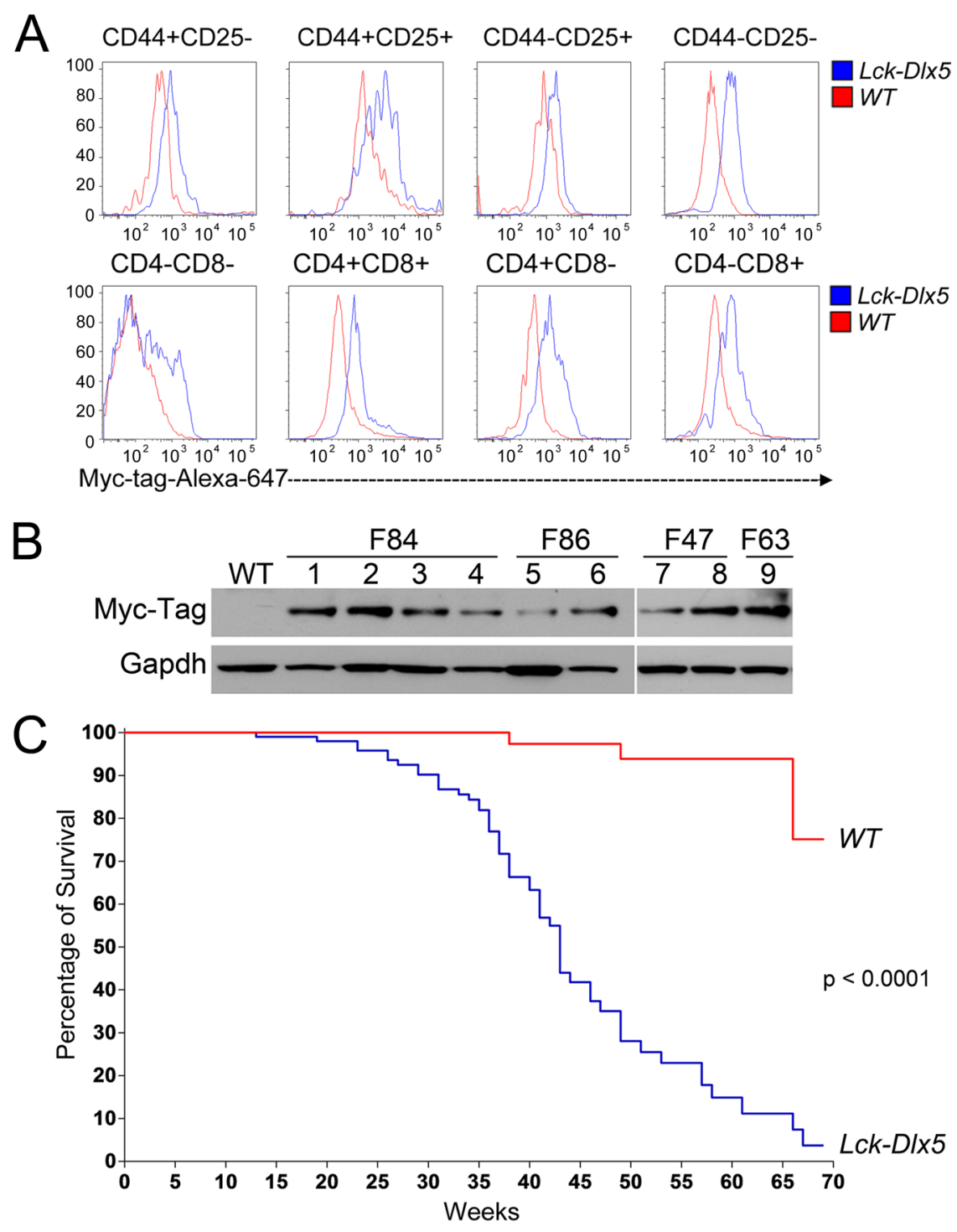

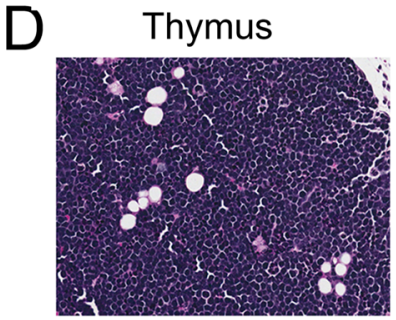

Kidney

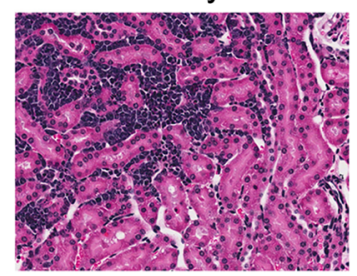

Lung

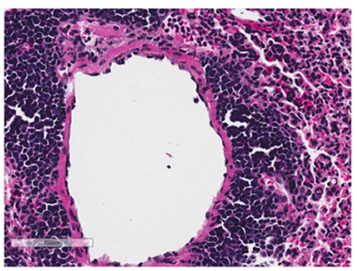

Spleen

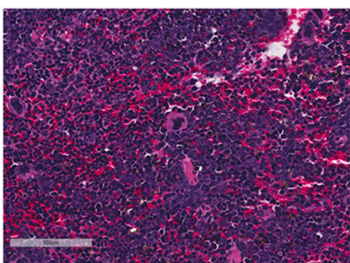

Liver

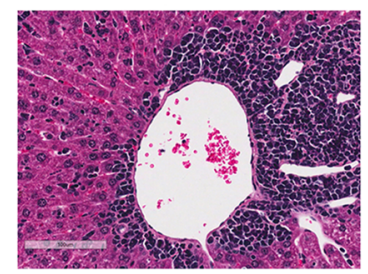

Bone Marrow

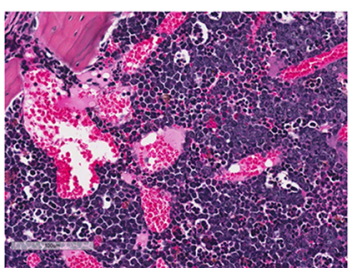

Figure 1: Lck-Dlx5 mice express Dlx5 throughout T-cell development and are predisposed to disseminated T-cell lymphomas. (A) representative flow cytometric analysis showing that Lck-driven, Myc-tag-labeled Dlx5 protein is expressed throughout thymic T-cell development. (B) expression of Myc-tagged Dlx 5 in thymic lymphomas from four transgenic founder lines (F84, F86 [mouse\# 801,793], F47 [mouse\# 0, 918], F63 [mouse\# 0]). (C) survival curve of Lck-Dlx5 transgenic mice from founder line F86. (D) H\&E staining showing representative T-cell lymphoma in thymus, lung, liver, kidney, spleen and marrow of Lck-Dlx5 mice. Bars $=100 \mu \mathrm{m}$. 
normal thymic T cells from WT mice (Figure 2A). To further discern factors potentially unique to Dlx5-driven lymphomagenesis, we compared gene expression profiles in lymphomas from Lck-Dlx 5 mice with those of LckMyrAkt2 animals. This comparison revealed enhanced expression of Notch and histone cluster genes specifically in Lck-Dlx5 lymphomas (Figure 2B). Sanger sequencing revealed that $\sim 60 \%$ of tumors in Lck-Dlx 5 mice exhibited activating mutations in HD and PEST domains of Notch1 (Supplementary Table 2). The commonly expressed genes in tumors from Lck-Dlx 5 mice and Lck-MyrAkt 2 mice, as well as uniquely expressed genes in those from Lck-Dlx5 animals, were further categorized. Notably, expression of Ptk2/Fak was upregulated in both Lck-Dlx5- and LckMyrAkt2-derived lymphomas (Supplementary Figure 2C). In contrast, aberrant expression of Dlx1as, and Notch were a specific feature of Lck-Dlx 5 lymphomas, suggesting that a Dlx5-Notch-Myc axis plays a critical role in the pathogenesis of these tumors (Supplementary Figure 2D). Real-time RT-PCR confirmed that Notch1, Notch3, Hes 1 and Myc mRNA levels were upregulated in Lck-Dlx5 lymphomas, when compared with those of WT thymic T-cells and Lck-MyrAkt2 lymphomas (Figure 2C). Interestingly, the only Lck-Dlx5 tumor (1/11) without overexpression of Notch1 and Notch3 instead showed upregulation of Notch2, further suggesting an essential role for aberrant Notch signaling in Dlx5-driven T-cell lymphomagenesis. Ccnd1/Cyclin D1 mRNA expression was also frequently elevated in Lck-Dlx5 tumors (Figure 2C). Moreover, immunoblotting demonstrated consistent upregulation of Notch1, Notch3, Myc, Hes1 and activation of Akt, as well as loss of Pten expression (Figure 2D; Supplementary Figure 2E). NIC1 specific antibody (Val1744) also revealed high levels of NIC1 in lymphoma cells from $L c k$-Dlx 5 mice, but no expression in tumors cells from Lck-MyrAkt2 mice. The truncated NIC1 proteins seen in cell lines F63-0 and F86-793 (Figure 2D) correspond with our Sanger sequencing data, which revealed nonsense mutations that were predicted to result in protein truncations (Supplementary Table 2). Notably, these features resemble the major molecular signature of human T-ALL. Immunohistochemistry also confirmed that Notch1, Notch3, Hes1, Myc and phospho (active)-Akt are upregulated in primary and metastatic lymphomas from Lck-Dlx5 mice (Figure 2E; Supplementary Figure 2F). Like human primary T-ALL, in which TP53 mutations are rare $[15,16]$, lymphomas from $L c k-D l x 5$ mice did not exhibit mutations in Tp53 (not shown).

To determine if the findings in our murine model have clinical relevance, we performed real-time PCR on 15 pediatric T-ALL specimens and found that 3 samples had high levels of expression of DLX5. Notably, these three samples also had elevated expression of NOTCH1, and in one case, NOTCH3 (Figure 2F). A search for published studies in expression repositories such as GEO and ArrayExpress websites revealed five studies of human
T-cell ALL or T-cell lymphoma containing $D L X 5$ expression data. We found that DLX5 was over-expressed (z-score $>1$ ) in $7-17 \%$ of cases in these studies, the exception being a smaller series by Iqbal et al. in which 2 of 5 cases expressed $D L X 5$. Approximately $4 \%$ of samples showed higher expression of DLX5 based on Z-score > 2 [3, 17-19].

\section{Dlx5 is required for tumor maintenance via activation of Notch and Akt}

To determine if Dlx 5 is still required after tumor initiation, knockdown of Dlx 5 in Lck-Dlx 5 tumor cells was achieved using retroviral-mediated expression of shRNA. Dlx5 knockdown resulted in reduced tumor cell viability/ proliferation (Figure 3A) and an increased population of apoptotic sub-G1 cells (Figure 3B; Supplementary Figure $3 \mathrm{~A}$ ) due, in part, to down regulation of phosphoAkt and/or Notch1/3 (Figure 3C). To test whether overexpression of active Notch or Akt could reverse the knockdown effect, cells with knockdown of Dlx5 were infected with retroviral NIC3 or MyrAkt2; cell viability was measured $3 \mathrm{~d}$ after transduction and the knockdown effect was rescued (Figure 3D; Supplementary Figure 3B). We next inoculated cells into immunocompromised NSG mice via subcutaneous injection, and tumor weight was assessed 2 weeks after injection. We found that Dlx 5 expression was required for tumor growth in vivo and that knockdown of Dlx 5 could be partially rescued by expression of either NIC3 or MyrAkt2 (Figure 3E, 3F).

\section{Activation of both Notch and Akt are essential for survival and proliferation of Lck-Dlx 5 lymphoma cells}

To directly block the Notch pathway, we transduced GFP-coupled Dominant negative mastermind ( $D N-M M)$ into Lck-Dlx 5 lymphoma cells, which resulted in reduced cell viability. Similarly, inhibition of the Akt pathway by re-introducing Pten expression reduced cell proliferation (Figure 4A, 4B). We next injected Lck-Dlx5 lymphoma cells into the tail vein of NSG mice. The Lck-Dlx5 lymphoma cells formed nodules primarily in liver and spleen 2 weeks after intravenous injection (Supplementary Figure 4A). Spleen and liver weights were measured to estimate lymphoma growth in these two organs. Inhibition of Akt or Notch signaling in Lck-Dlx 5 lymphoma cells by transduction of Pten or $D N-M M$, respectively, markedly suppressed tumor growth in vivo (Figure 4C).

Previous studies have demonstrated that the majority of human T-ALL cell lines are resistant to $\gamma$-secretase inhibitors due largely to mutations of PTEN or FBXW7 $[20,21]$. Interestingly, although expression of Pten protein was absent in Lck-Dlx 5 lymphoma cells, these cells were still sensitive to Notch inhibitors. Treatment with DAPT or $\gamma$-secretase inhibitor XXI for 2 days strongly suppressed the proliferation of $L c k-D l x 5$ lymphoma cells, as shown by 

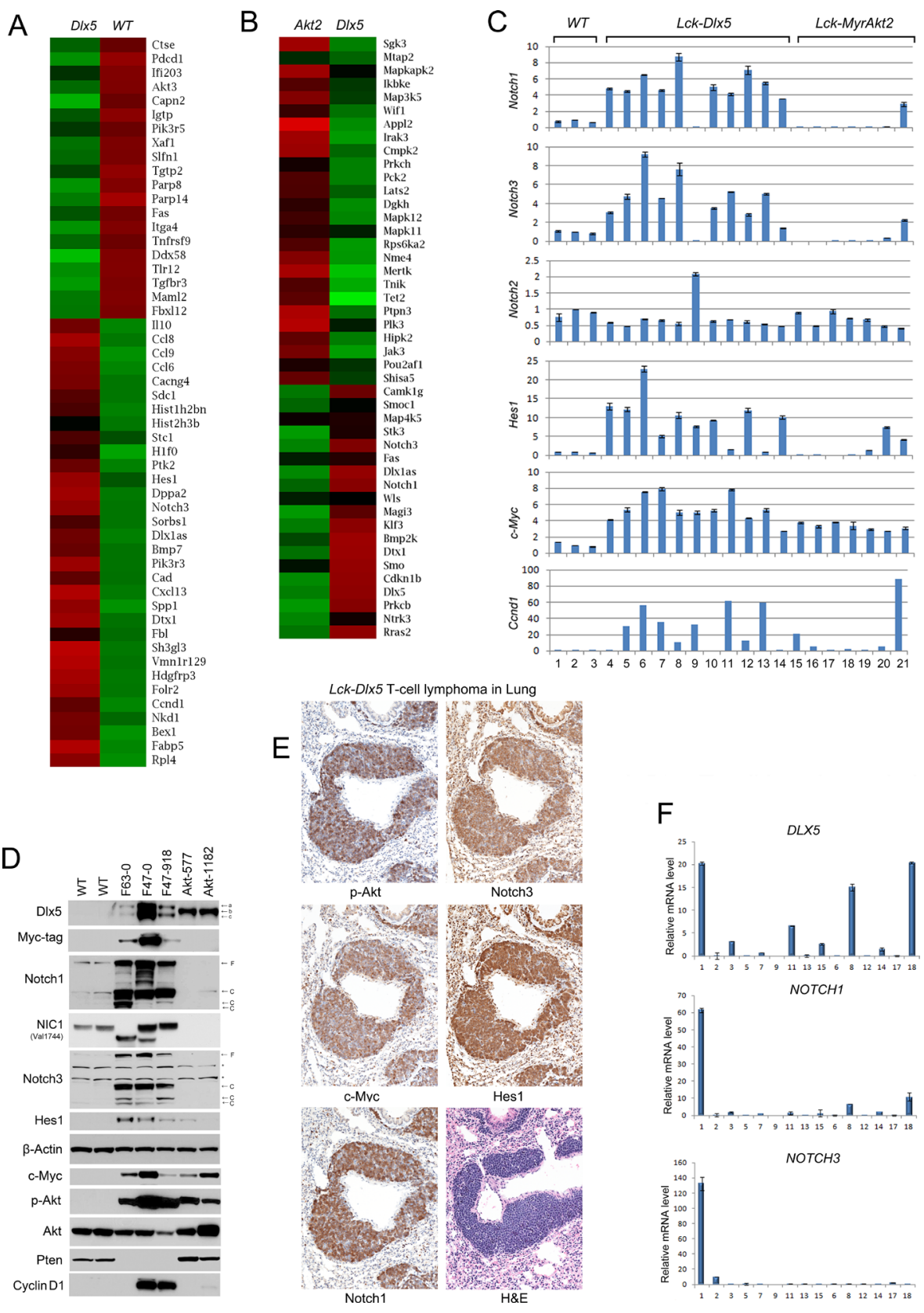

Figure 2: Lck-Dlx5 lymphomas exhibit a molecular signature reminiscent of human T-ALL. (A) RNA microarray heatmap demonstrating that compared to thymocytes from three WT mice, primary T-cell lymphomas from three Lck-Dlx5 mice exhibit upregulation (Red) of genes involved in Notch signaling, cell cycle progression, chromatin modification, calcium homeostasis, glucose, fatty acid and nucleotide metabolism, as well as classical Dlx5 target genes. Down-regulated genes (Green) are implicated in pro-apoptotic events (Tnf, Fas, Xaf1, INFb signaling-related genes), tumor suppression, and DNA damage repair. (B) compared to T-cell lymphomas from LckMyrAkt2 mice, Lck-Dlx5 lymphomas show upregulation of Notch1/3 and downregulation of Lats2, Appl2, Jak3, Tet2, and Tnik. Note that in the interests of journal space, the heatmap shown here includes only one sample from each group. The full heatmaps for all three samples examined per group can be found in Supplementary Figure 2C. (C) real-time PCR on three T-cell samples from WT mice (samples 1-3), 11 primary lymphomas from Lck-Dlx5 mice (samples 4-14: F86-785, -801, -793, -1149; F63-0, -1263, -1210; F47-0, -1247, -918; and F84-1063), and 7 lymphomas from Lck-MyrAkt2 mice (samples 15-21: F72-918, -2811, -3148, -3154; and F420-577, -1174, -1073), confirming unique upregulation of Notch signaling in Dlx5-driven tumors. Data $=$ mean \pm SEM. (D) immunoblot showing overexpression of Notch1, NIC1, Notch3, Hes1 and Myc in lymphoma lines (F63-0, F47-0, F47-918) from Lck-Dlx5 mice. Loss of Pten expression and corresponding high levels of phospho-Akt in $L c k$-Dlx5-derived tumor cells are also shown, as are cyclin D1 levels. Dlx5 proteins have high molecular weight $(\mathrm{a}, \mathrm{b})$ and low molecular weight (c) forms. Notch proteins have full length (F) and cleaved (C) forms. *, non-specific bands. (E) H\&E and immunohistochemical staining of lymphoma invading lung of Lck-Dlx5 mouse. Note strong staining for Notch1, Notch3, Hes1, Myc and phospho-Akt in lymphoma. (F) summary of real-time PCR analysis of RNA from 15 pediatric T-ALL specimens showing expression levels of DLX5, NOTCH1 and NOTCH3. 
cell cycle analysis (Figure 4D; Supplementary Figure 4B). Moreover, immunoblotting revealed that Notch signaling is diminished following treatment with $\gamma$-secretase inhibitors (Figure 4E, 4F). The mTOR inhibitor RAD001 enhanced the inhibitory role of Notch inhibitors, as demonstrated by a further reduction in cell viability (Supplementary Figure 4C) and cell cycle arrest (Supplementary Figure 4D, 4E). When combined with XXI or DAPT, RAD001 strongly inhibited expression of Cyclin A and Cyclin D1 (Supplementary Figure 4F). Additionally, the Akt inhibitor GSK690693 induced apoptotic sub-G1 DNA condensation within 20 h (Figure 4G; Supplementary Figure 4G), which was also accompanied by activation of caspase-3 (Figure 4H), consistent with Akt's pro-survival role being independent of mTOR. Moreover, combinational treatment of Lck-Dlx5 tumor cells with GSK690693 and DAPT or $\gamma$-secretase inhibitor XXI resulted in a marked increase in cell death as shown by augmentation of the sub-G1 peak and by activation of caspase-3 (Figure 4I, 4J; Supplementary Figure 4H). Collectively, these findings indicate that activation of the Akt and Notch pathways contribute independently to Dlx5-mediated oncogenesis.

\section{Notch1 and Notch3 are direct targets of Dlx5}

ChIP-seq was used to discover Dlx5 target genes. Dlx5 was found to bind to promoter or enhancer regions of various genes, including Notch1, Notch3, Irs2 and Ccnd1
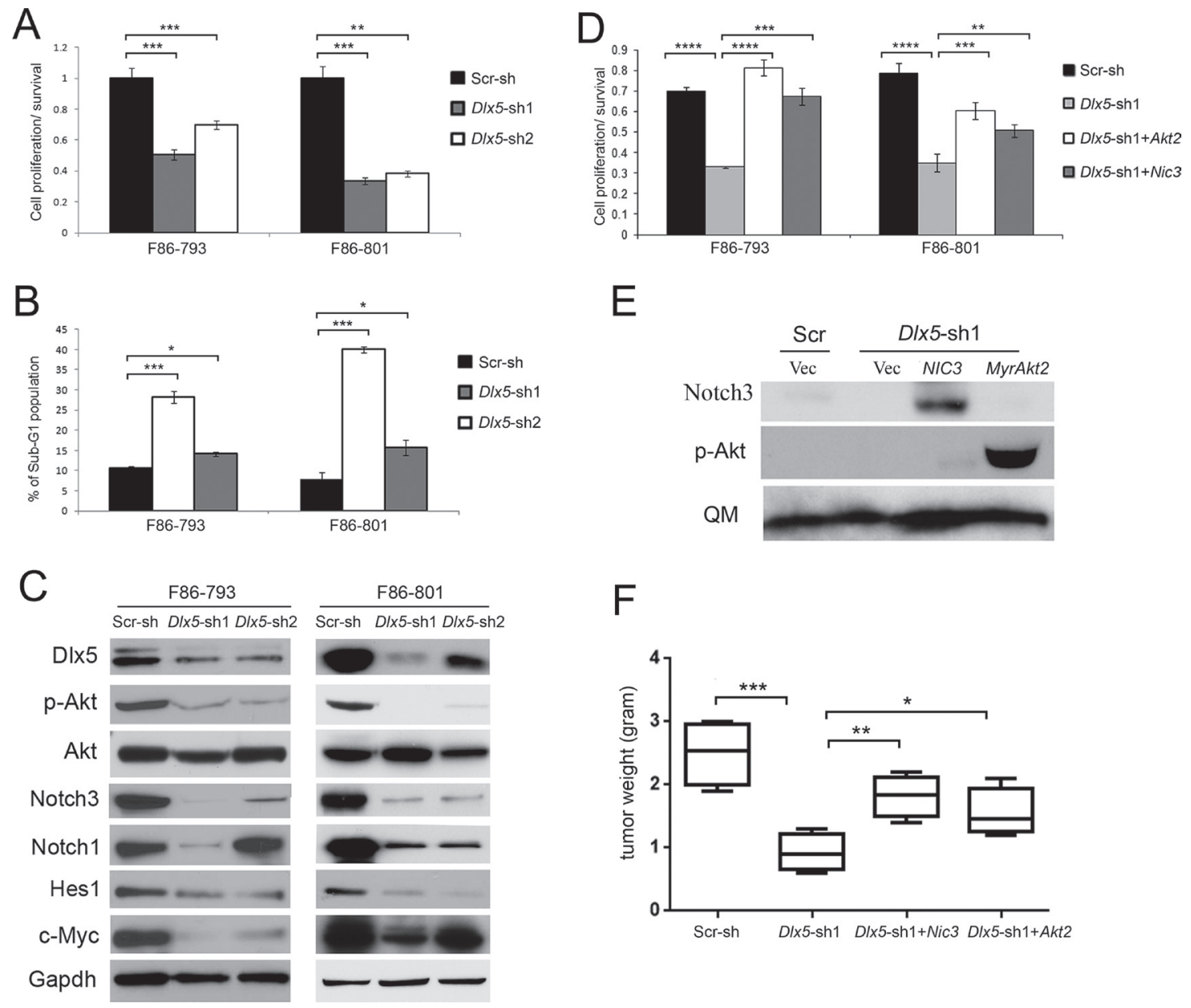

Figure 3: Dlx 5 is required for survival of $\boldsymbol{L} \boldsymbol{c k}$-Dlx 5 lymphoma cells. (A) lymphoma cells from $L c k$-Dlx 5 mice were transduced with two different retrovirus-mediated shRNAs against $D l x 5$, or with scrambled control shRNA (Scr). Tumor cell viability was reduced after $D l x 5$ knockdown as shown by MTS assay. (B) Flow cytometry analysis depicting increased sub-G1 apoptotic population in lymphoma cells transduced with Dlx5 shRNA. (C) immunoblotting revealing diminished full-length Notch1, Notch3, Myc, Hes1, and phosphoAkt levels following Dlx5 knockdown. (D) MTS assay demonstrating rescue of Dlx5 knockdown cells by retrovirus-mediated ectopic expression of active Notch3 (NIC3) or activated Akt2 (MyrAkt2). (E) immunoblot analysis depicting re-expression of active forms of Notch3 and Akt2 in F86-793 line (Note: MSCV-mediated transduction delivers high expression of genes encoding Notch3 or constitutively activated Akt2, which required only very short film exposure times; thus, the relatively much weaker endogenous Notch3 or p-Akt are not discernable here). (F) decreased growth of Dlx 5 knockdown cells is partially rescued by ectopic expression of NIC3 or activated Akt2. Cells were inoculated subcutaneously into NSG mice, and tumor weights were measured after 2 weeks. Significance: $* p<0.05 ; * * p<0.01$; $* * * p<0.005 ; * * * * p<0.001 ; * * * * * p<0.0005$. 


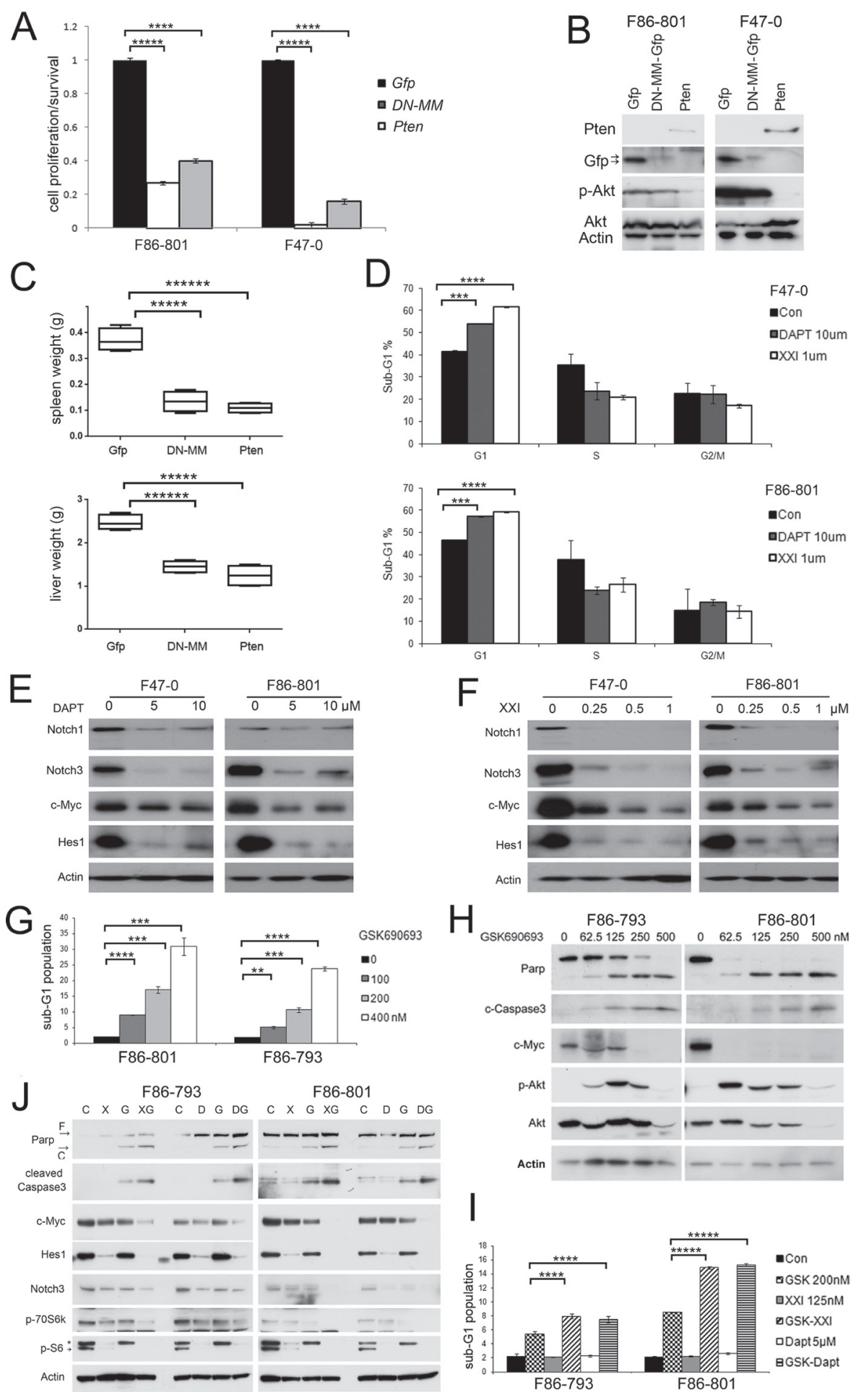

Figure 4: Akt and Notch signaling are essential for proliferation and survival of Lck-Dlx 5 lymphoma cells. (A) ectopic expression of Pten or dominant-negative mastermind (DNMM) inhibits survival of $L c k$-Dlx 5 tumor cells as shown by MTS assay. Control was ectopically expressed $G f p$. (B) immunoblot analysis depicting expression of exogenous $D N M M$-Gfp and Pten. (C) tumor growth of tail vein-injected Lck-Dlx5 lymphoma cells into NSG mice. Weights of tumor cell-infiltrated liver and spleen are shown. (D) flow cytometry showing that Notch inhibitors XXI or DAPT suppress cell cycle progression of $L c k$-Dlx 5 tumor cells. (E and $\mathbf{F}$ ) Notch inhibitors decrease Notch1, Notch3, Hes1 and c-Myc protein levels. Cells were harvested $24 \mathrm{~h}$ after beginning treatment with inhibitor. (G and $\mathbf{H})$ Akt inhibitor GSK690693 alone triggers sub-G1 DNA condensation, caspase 3 activation, and Parp cleavage in Lck-Dlx5 tumor cells. Upregulation of phospho-Akt in GSK690693-treated cells is indicative of a feedback loop to Akt, although downstream effectors of Akt signaling, e.g., p-mTOR and p-p70S6k are downregulated [44]. (I and J) when cells were treated with GSK690693 plus Notch inhibitors XXI or DAPT, apoptosis was significantly increased, as assessed by flow cytometry and immunoblotting. *, residual Hes1 band due to previous blotting with Hes1 antibody. Significance: ${ }^{*} p<0.05 ; * * p<0.01 ; * * *<0.005 ; * * * * p<0.001 ; * * * * p<0.0005$. 
(Figure 5A, Supplementary Figure 5A). Multiple Em for Motif Elicitation (MEME) analysis revealed that the majority of Dlx 5 consensus binding belongs to the classic binding motif (TAATT) of the Dlx family, which served as an internal quality control for our ChIP-Seq experiment (Figure 5B). BLAST analysis demonstrated that the Dlx5 binding consensus on the Irs 2 promoters is conserved among human, monkey, rat and mouse (Figure 5C). ChIPqPCR confirmed that Dlx5 specifically binds to Notch1 and Notch3 enhancer elements (Figure 5D, 5E). The mouse Notch 1 and Notch 3 promoter fragments were sequentially cloned into pGL3 vector. Two enhancer elements downstream of the Notch1 gene were inserted separately into pGL3 downstream of the Notch1 core promoter sequence (N1P1) at a SalI site within the vector (Figure $5 F)$. In addition, the enhancer element located in intron 2 of Notch3, downstream of a 600-bp promoter fragment containing the core promoter (N3P1), was also cloned into pGL3. HEK293T cells were then co-transfected with pGL3-Notch1 promoter/enhancers or Notch3 promoter/ enhancer and WT or mutant Dlx5 plasmids. Luciferase activity was measured $16 \mathrm{~h}$ after transfection. The reporter assay demonstrated that wild-type Dlx5, but not a Dlx5 mutant missing the DNA binding domain, augments transcription of the Notch1 promoter in the presence of Notch1 enhancer A or B (Figure 5G). Likewise, Dlx5 expression enhanced the luciferase activity of the Notch 3 promoter when its enhancer was inserted into the same plasmid (Figure $5 \mathrm{H}$ ). Interestingly, other members of the Dlx family, i.e., Dlx4 and Dlx6, also were capable of upregulating Notch1 and Notch3 luciferase activity via their enhancers (Supplementary Figure 5B, 5C).

\section{Dlx5 induces early alteration of Notch and Akt signaling during T-cell development}

Real-time PCR showed that in 5-week-old mice, Notch1 and Notch3 mRNAs are upregulated in normal thymocytes from Lck-Dlx5 mice when compared to those of normal thymic $\mathrm{T}$ cells from agematched WT littermates or Lck-MyrAkt2 mice, and their expression was further augmented in lymphomas from Lck-Dlx5 mice (Figure 6A). In contrast, Notch2 and Notch 4 mRNAs were not altered in the tested samples (Supplementary Figure 6A). Hes 1 and Myc, two target genes of Notch signaling, also showed elevated mRNA levels (Supplementary Figure 6B). Additionally, transcript levels of Irs 2 and $C c n d 1$, two genes identified by ChIP-seq analysis as Dlx 5 targets, also were upregulated (Figure 6B; Supplementary Figure 6B). Immunoblotting confirmed that normal thymic $\mathrm{T}$ cells from Lck-Dlx 5 mice have elevated Notch $1 / 3$ protein expression compared to that of normal thymic $\mathrm{T}$ cells from WT mice. Further upregulation of Notch $1 / 3$ protein levels was observed in Lck-Dlx 5 lymphoma cells (Figure 6C). The reason why mRNA levels of Notch1 and Notch3 were 2-4 fold greater in lymphoma cells than in normal T cells from $L c k-D l x 5$ mice was not due to gene amplification, as array-CGH analysis revealed no change of Notch1/3 gene copy number. Instead, the increased gene transcript levels in tumor cells appeared to result from increased interaction between Notch promoters and Dlx5-bound Notch enhancers, as revealed by CCC assay (Figure 6D, 6E). Furthermore, flow cytometry analysis revealed that Notch $1 / 3$ protein levels are elevated early on during T-cell development from CD4-CD8- Double-Negative (DN1 [CD25- CD44+]; DN2[CD25+ CD44+]; DN3 [CD25+ CD44-]; and DN4 [CD25- CD44-]) stages (Figure 6F, 6G; Supplementary Figure 6C). Importantly, the increased full-length Notch protein levels resulted in elevated Notch cleavage (Supplementary Figure 6D). Hes1 and Myc proteins, targets of active Notch, were also upregulated in normal $\mathrm{T}$ cells from $L c k$-Dlx 5 mice (Figure 6C; Supplementary Figure $6 \mathrm{E}, 6 \mathrm{~F}$ ). This finding suggests that upregulation of Notch $1 / 3$ might serve as the first cooperating oncogenic "hit" in T-cell lymphomagenesis in Lck-Dlx 5 mice.

Interestingly, phospho-Akt levels were also consistently elevated in normal $\mathrm{T}$ cells from $L c k-D l x 5$ mice (Figure 6C). This increase was found to occur at the early stage of T-cell development from DN1 to DN4, CD4/CD8 DP and CD4-positive cells, but not in CD8positive cells (Figure 6H; Supplementary Figure 6G). We previously reported that in human ovarian carcinoma cells, DLX5 transactivates the IRS2 gene via direct binding to the IRS2 promoter, thereby resulting in enhanced AKT signaling. Similar findings were documented in T cells from Lck-Dlx5 mice, based on ChIP-seq analysis (Figure 5A). We found that Irs2 transcript and protein levels are increased in non-malignant $\mathrm{T}$ cells from $L c k$ $D l x 5$ mice, but not in their malignant T-cell counterparts that have lost expression of Pten protein (Figure 6B, 6C). Loss of Pten expression results in constitutive activation of Akt, circumventing the need for upregulation of Irs2. Thus, it appears that Irs2-related Akt activation is a driving force from the very beginning of Dlx5-provoked T-cell lymphomagenesis, with more profound activation due to Pten loss occurring in fully malignant cells.

\section{Dlx 5 promotes $T$ cell survival during b-selection via Notch and Akt}

Immunoblot analysis revealed that non-malignant thymic $\mathrm{T}$ cells from $L c k$-Dlx 5 mice have significantly less activation of caspase-3 than tumor cells (Figure 6C; Supplementary Figure 7A). Flow cytometry analysis showed that Lck-Dlx5 mice have less CD4+CD8+ DP cells, with more cells at the DN stage (Figure 7A). This is likely due to the fact that the DN population in $L c k$ Dlx 5 mice has increased viability, consistent with reduced AnnexinV/PI staining (Figure 7B; Supplementary Figure 7B). Moreover, the DN population in Lck-Dlx5 mice had less caspase-3 activation (Supplementary 
A
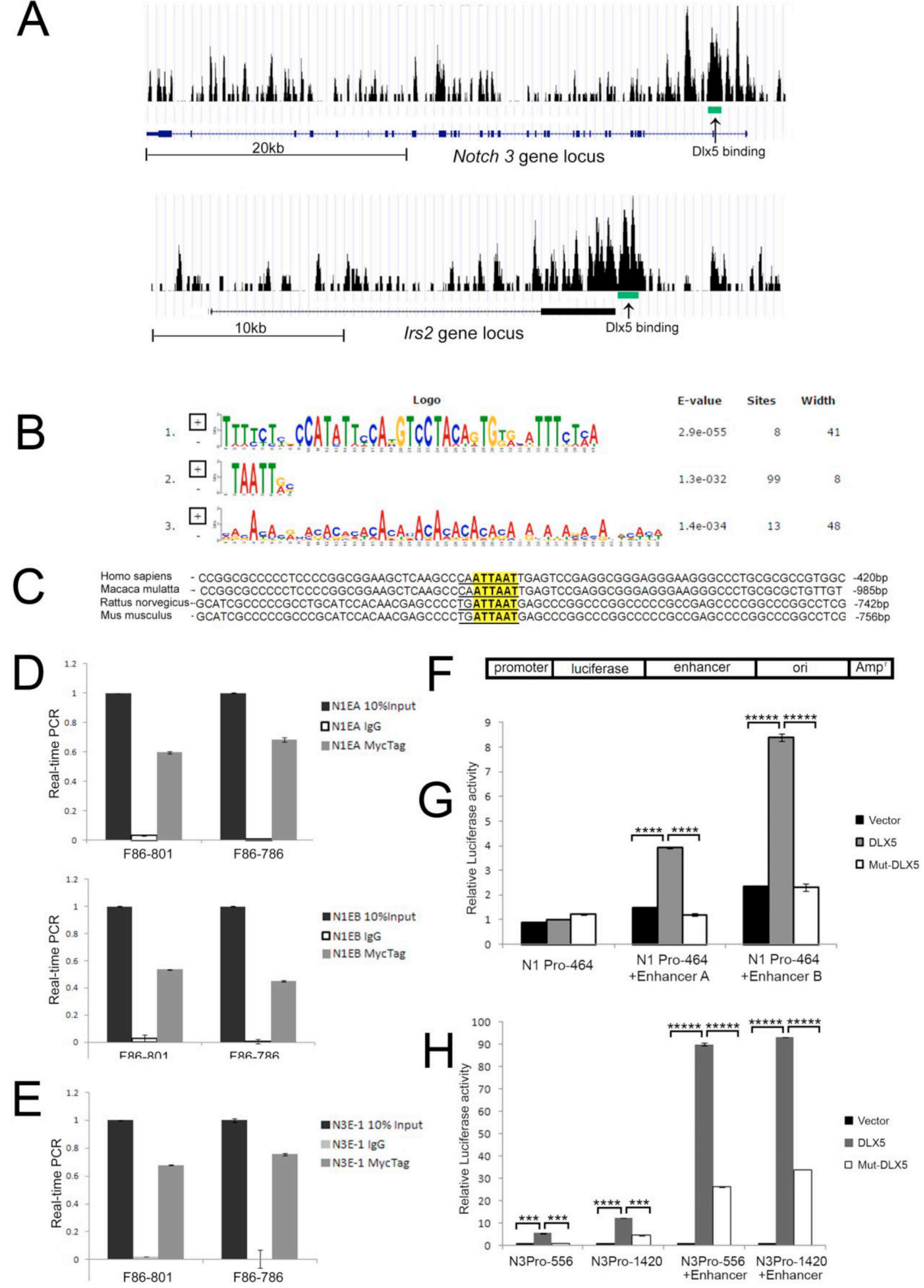

Figure 5: Notch1 and Notch3 are direct downstream targets of Dlx5. (A) ChIP-seq analysis revealed Dlx5 binding sequences in Notch 3 (MACS $p$ value: $-10 *$ LOG10 $=70.54$ ) and Irs2 (MACS $p$ value: $-10 *$ LOG10 $=76.57$ ) loci as depicted in screenshots from UCSC Genome Browser. Arrows point to binding intervals in each locus. (B) Multiple Em for Motif Elicitation (MEME) shows that the majority of the Dlx5 binding consensus site belongs to the classic binding motif (TAATT) of the Dlx family. (C) Irs2 promoters from human, monkey, rat and mouse contain Dlx5 binding consensus. (D) results of ChIP-qPCR assay confirmed that Dlx5 binds to two enhancer elements at end of Notch1 gene. (E) ChIP-qPCR verified that Dlx5 binds to enhancer element located in Notch3 intron 2. (F) schematic diagram of Notch 1 and Notch 3 promoter/enhancer reporter plasmids. Luciferase assay confirmed that Dlx 5 , but not Dlx 5 mutant lacking the DNA binding domain, activates Notch1 $(\mathbf{G})$ and $\operatorname{Notch} 3(\mathbf{H})$ promoters upon binding to their respective enhancer elements. 

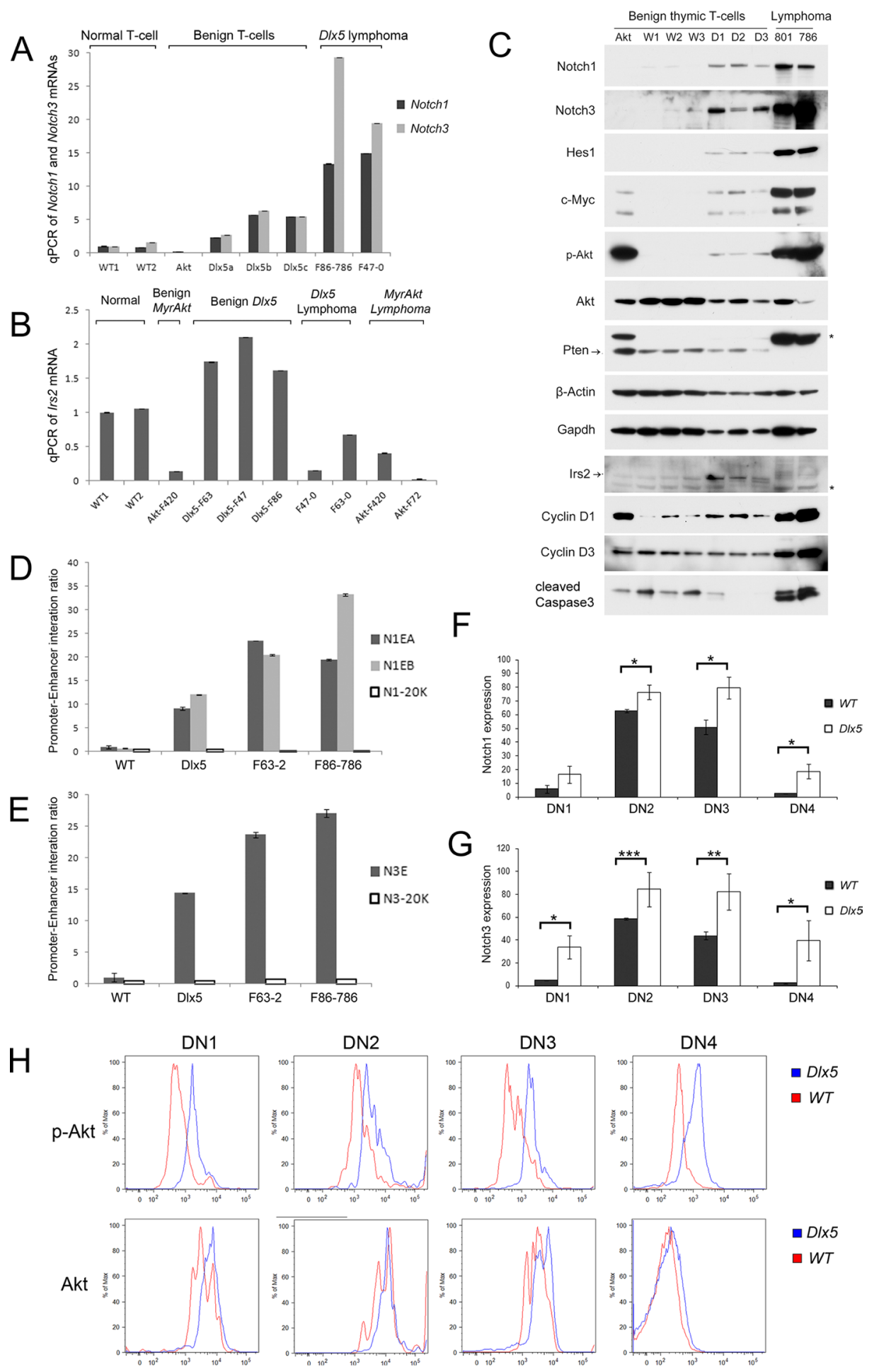

- DIX5

$\square T$

Alexa 488

Figure 6: Dlx5 induces activation of Notch and Akt during T-cell development. Real-time PCR was performed on thymic T cells from 5-week-old Lck-Dlx5 mice (D1, D2, D3) prior to tumor development, WT littermates (W1,W2,W3), a 5-week-old LckMyrAkt2 mouse (Akt), and Lck-Dlx5 lymphomas $(801,793)$ to compare expression levels of full-length Notchl/3 (A) and Irs2 (B). (C) immunoblotting confirming increased expression of Notch $1 / 3$ in the non-malignant $\mathrm{T}$ cells from $L c k-D l x 5$ mice, with further enhanced expression in tumors from these mice. (D and E) CCC assay showing frequency of interaction of Dlx5-bound Notch1 enhancers to Notch1 promoter (D) and Dlx5-bound Notch3 enhancer to Notch3 promoter (E). N1-20K and N3-20K refer to control DNA segments $20 \mathrm{~Kb}$ downstream of Notch1 or Notch 3 loci, respectively. (F and $\mathbf{G}$ ) Flow cytometry analysis of various stages of T-cell development showing elevated expression of Notch1 (F) and Notch3 (G) in all DN stages (DN1-DN4) in Lck-Dlx5 mice compared to that of WT mice. (H) Flow cytometry analysis revealing increased expression of activated Akt kinase (p-Akt) in all DN stages in T cells from Lck-Dlx 5 mice. 
Figure 7C, 7D). CD44 and CD25 staining demonstrated that $L c k-D l x 5$ mice have an increased $T$ cell population at the CD44-CD25+ DN3 stage (Figure 7C), and AnnexinV/ PI staining indicated augmented cell survival at this stage (Figure 7D). Furthermore, DAPT treatment for $5 \mathrm{~d}$ reversed the enhanced viability of the $\mathrm{DN} 3$ cell population in Lck-Dlx 5 mice (Figure 7D; Supplementary Figure 7E), consistent with caspase-3 activation in DN3 cells (Figure 7E; Supplementary Figure 7F). Nevertheless, treatment of $L c k-D l x 5$ mice with DAPT or RAD001 resulted in increased gross cleavage of caspase- 3 in thymic $\mathrm{T}$ cells (Figure 7F). The mechanism of Dlx5-induced T-cell lymphomagenesis is summarized in Figure 7G.

\section{DISCUSSION}

Cancer may be considered as development gone awry. Multiple homeobox genes have been implicated in tumor formation. Chromosomal rearrangements with breakpoints in TCR genes are common in T-cell malignancies. These rearrangements place TCR regulatory sequences beside T-cell-related transcription factor genes, leading to their oncogenic activation. For example, one recurrent rearrangement, inversion of chromosome 7, $\operatorname{inv}(7)(p 15 q 34)$, is observed in a subset of patients with T-ALL or T-NHL $[3,22]$. This rearrangement repositions the HOXA gene on chromosome band $7 \mathrm{p} 15$ adjacent the $T R B$ locus at $7 \mathrm{q} 34$, which is analogous to the inversion inv(6)(A2B1) we reported in T-cell lymphomas from $L c k$ MyrAkt2 mice [6].

Little is known about the target genes of homeoproteins, although transfection studies with HOXA10 revealed upregulation of several genes encoding components of the Wnt pathway, including $W N T 10 B$ and FZD1, which are essential in hematopoietic stem cell renewal [23]. Notably, Soulier and colleagues determined that the HOXA-related subgroup of T-ALL is characterized by high levels of expression of several genes known to be critical for T-cell differentiation and/or oncogenesis, including NOTCH3 [3], which we found to be transcriptionally regulated by Dlx 5 in lymphomas from Lck-Dlx5 mice. Interestingly, two of the four HOXA10-rearranged cases also had prototypical NOTCH1 mutations, suggesting that HOXA10 and NOTCH1 abnormalities cooperate oncogenically in such T-ALLs. Notably, $60 \%$ of tumors from our LckDlx 5 mice also harbored activating mutations of Notch1. During hematopoietic stem cell differentiation, Notch1 drives the transition of lymphoid progenitor cells toward T lymphocytes [24], and Notch1 is considered a master regulator of T-cell fate specification [25]. Moreover, Notch signaling promotes survival of pre-T cells at the b-selection checkpoint by regulating cell metabolism [26]. In human T-ALL, NOTCH1 is targeted by activating mutations in $50-70 \%$ of cases $[14,27]$. Collectively, these clinical data, like our experimental findings in $L c k-D l x 5$ mice, are consistent with a model in which developmental homeobox genes play a central role in T-ALL oncogenesis.

Pear et al. demonstrated that intracellular Notch1 (ICN1) could directly drive murine T-cell lymphoma formation [28]. However, most gain-of-function NOTCH1 mutations found in human T-ALL do not generate downstream signals of sufficient strength to efficiently initiate leukemia development in mice [29]. Nevertheless, such weak gain-of-function $\mathrm{NOTCH} 1$ alleles were able to hasten the onset of T-cell malignancy initiated by other leukemogenic events, such as activation of K-ras. Unlike NOTCH1, NOTCH3 mutations have not been reported in human T-ALL, although NOTCH3 is often highly expressed [30]. It is also notable that although Dlx5 was expressed in the two Lck-MyrAkt2 lymphoma cell lines shown in Figure 2C, Notch1 and Notch3 levels were not upregulated. Moreover, as shown in Figure 2C, among three Lck-MyrAkt2 cell lines that expressed Dlx5 (lines 19-21), real-time PCR analysis revealed transcriptional upregulation of Notchl/3 in only one (line 21). One possible reason for this is that a Notch $1 / 3$ enhancerspecific coactivator generally may be lacking in $L c k$ MyrAkt2 lymphoma cell lines expressing Dlx5. In any case, the collective findings indicate that $L c k$-Dlx 5 -driven lymphomagenesis differs from that of Lck-MyrAkt2-driven thymic lymphoma formation. Specifically, in Lck-MyrAkt2 mice, constitutively active Akt2 alone may serve as such a potent oncogenic factor that Myc activation, either directly via a $M y c$ translocation or indirectly through its transcriptional activation by Dlx5, is sufficient as a "second hit." In contrast, in $L c k$-Dlx 5 mice, which have a longer tumor latency than Lck-MyrAkt2 mice, multiple oncogenic factors, including Notch $1 / 3$, Irs2, Cyclin D, and Myc, appear to be required to initiate tumor formation.

Recently, NOTCH1 was identified as a direct target of DLX5 during squamous cell differentiation [31]. Using combinatorial unbiased approaches entailing ChIP-seq and microarray analysis, we determined that Dlx 5 binds to both the Notch1 and Notch 3 loci and promotes their transcription. Lck-Dlx 5 transgenic mice show upregulation of Notch1 and Notch3 throughout T-cell differentiation, and basal levels of Notch $1 / 3$ were further augmented in malignant T-cells due to increased enhancer-promoter interaction. Moreover, thymic T cells from $L c k-D l x 5$ mice were resistant to apoptosis during b-selection at the DN3 stage, and such resistance could be reversed by treatment with $\gamma$-secretase inhibitors. Thus, we concluded that Dlx5 directly transactivates Notch $1 / 3$ genes and promotes $\beta$-selection escape, which eventually results in tumor formation (Figure 7G). While lymphoma cells from $L c k$ Dlx 5 mice were sensitive to $\gamma$-secretase inhibitors, those from Lck-MyrAkt 2 mice (most of which did not show over expression of Notch1/3; Figure 2) were resistant, further strengthening our contention that Notch signaling is a driving force in Dlx5-mediated lymphomagenesis. Our study of human pediatric T-ALL revealed overexpression 

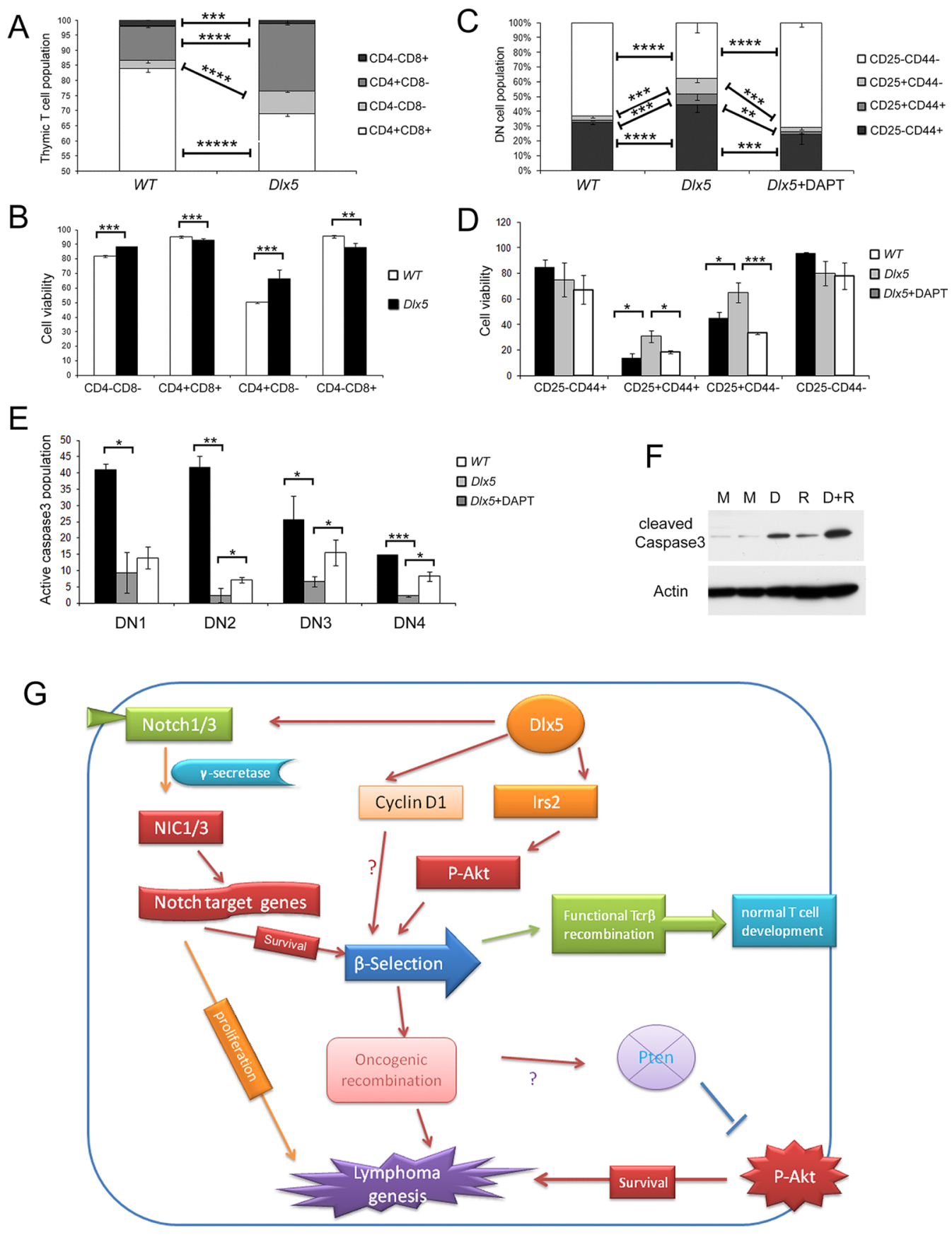

Figure 7: Dlx5 conveys survival signals via Notch and Akt during $\beta$-selection. (A) Flow cytometry analysis of thymic $\mathrm{T}$ cells from 5-week-old WT and $L c k$-Dlx 5 mice demonstrating that $L c k$-Dlx 5 mice have increased percentage of CD4-CD8- cells and decreased percentage of CD4+CD8+ cells. (B) modest but statistically significantly increased survival of CD4-CD8- (DN) T cells from Lck-Dlx5 mice, as shown by Annexin V/PI staining. (C) CD25 and CD44 staining showing increased population of CD25+CD44- cells in thymus of Lck-Dlx 5 mice. DN1 = CD25- CD44+; DN2 = CD25+ CD44+; DN3 = CD25+CD44-; and DN4 = CD25- CD44-. Administration of DAPT for $5 \mathrm{~d}$ resulted in a decrease in this cell population. (D) annexin V/PI staining demonstrating that CD25+CD44- population in $L c k$ Dlx 5 mice has increased cell viability compared to that of DN3 cells from WT mice, and that DAPT treatment reverses Dlx5's pro-survival effect. (E) staining for cleaved caspase3 showing that T cells from Lck-Dlx 5 mice have much less caspase3 activation at the DN3 stage compared to that of T cells from WT mice, which was partially rescued by treatment with DAPT. (F) immunoblot analysis demonstrating that treatment of T cells from $L c k$-Dlx 5 mice with DAPT (D) and/or RAD001 (R) results in increased gross cleavage of caspase3. M = mock (placebo) treatment. (G) proposed mechanism of Dlx5-induced lymphomagenesis. Aberrant expression of Dlx 5 in immature thymic $\mathrm{T}$ cells results in direct binding to Notchl and Notch3 enhancer sequences as well as to promoters of Irs 2 and $C c n d 1$, which activates expression of these critical target genes. Resulting augmented Notch and Akt signaling promotes survival of affected immature $\mathrm{T}$ cells during $\beta$-selection. Fully transformed $\mathrm{T}$ cells continue to rely on Notch and Akt signaling for proliferation and dissemination via subsequent mutation of Notch1 and loss of Pten. 
of DLX5 in about $20 \%$ cases, with co-upregulation of NOTCH1 and, to a lesser extent, NOTCH3. Intriguingly, not all homeobox genes behave in a similar manner, as a recent report showed that TLX1 actually inhibits $N O T C H 3$ expression [32].

During T-cell development, IL-7 induces a much greater increase in Pi3k-Akt activity via Irs2 than by Irs1, suggesting an important role for Irs 2 in T-cell differentiation [33]. Moreover, Irs2-deficient $\mathrm{T}$ cells show defects in IL-4-induced proliferation and differentiation in mice [34]. Recent studies demonstrated that $\sim 10 \%$ of T-ALL specimens display activating $I L 7 R$ mutations resulting constitutive activation of JAK/STAT5 and PI3K/Akt/mTOR pathways [35, 36]. Interestingly, our ChIP-seq studies revealed that Irs 2 is a direct target of Dlx5, and Lck-Dlx5 mice exhibit elevated Akt signaling beginning early in T-cell differentiation via enhanced expression of Irs2, which would be expected to augment Akt activity and facilitate Notch-induced lymphomagenesis.

AKT signaling is frequently activated $(\sim 50 \%)$ in human T-ALLs, mostly due to alterations of PTEN, $P I 3 K$, and $A K T$ genes $[37,38]$. We previously reported that DLX5 contributes to AKT signaling in human ovarian cancer cells via direct upregulation of IRS2 transcription [11]. When overexpressed, IRS2 has been shown to cause mammary tumorigenesis and metastasis [39]. In this investigation, we found that all lymphomas from $L c k-D l x 5$ mice have loss of Pten protein expression due to mutation/deletion or epigenetic silencing [13], resulting in constitutive activation of Akt. Thus, in both Lck-MyrAkt2 mice and Lck-Dlx5 mice, tumor formation appears to require cooperation between Akt and Dlx 5 or Myc. In Lck-MyrAkt 2 mice, we proposed that activation of the Akt pathway acts as the initial "hit" to promote cell survival and genomic instability, whereas the acquisition of T-cell-specific overexpression of Dlx5 leads to lymphomagenesis [40]. In Lck-Dlx5 mice, on the other hand, Akt activation due to Pten loss may act as a second hit during Dlx5-driven $\mathrm{T}$ cell lymphomagenesis.

In summary, our studies show that Dlx5 initiates T-cell lymphomagenesis via direct binding to Notch $1 / 3$ and Irs 2 genes to augment their transcription, which switches on Notch and Akt signaling, respectively, during T-cell fate decisions. The resulting spontaneous T-cell lymphomas maintain addiction to Dlx 5 through further intensified Notch and Akt signaling, thus connecting the Dlx5 homeoprotein to two pathways that are also known to be critically involved in the proliferation and survival, respectively, of human T-ALL. More generally, these experimental findings provide mechanistic insights about how transcriptional reactivation of a homeobox gene can drive T-ALL development via epigenetic reprogramming.

\section{MATERIALS AND METHODS}

\section{Primary tumors and tumor-derived cell lines}

$L c k-D l x 5$ mice were examined daily and sacrificed upon evidence of difficulty in breathing, severe weight loss, or when tumor burden is otherwise obvious, under IACUC approval in accordance with NIH guidelines. At the time of death due to lymphoma development, a portion of each tumor was snap-frozen in liquid nitrogen, another portion was kept in Trizol for Microarray, and the remaining portion was placed into culture in an attempt to establish tumor cell lines for subsequent Chip-Seq, immunoblotting, and MTS assays as well as for in vitro studies with pathway inhibitors. For microarray expression analysis and realtime PCR, primary tumor samples were used.

\section{Reagents}

Antibodies against Dlx5, Notch3, cyclin A, cyclin D1, Irs2, Gapdh and $\beta$-actin were from Santa Cruz Biotechnology; antibodies against Notch1, Notch1 (Val1744), Pten, p-Akt, Akt, Myc-Tag, Myc-TagAlexa647, and cleaved caspase-3-Alexa488 were from Cell Signaling. Akt pathway inhibitors GSK690693 and RAD001 were from Selleckchem (Houston, TX). Hes1 luciferase vector and Notch3 plasmid were from AddGene. MSCV-MyrAkt2 plasmid was as described [11], and MSCV-GFP-DN-MM plasmid was kindly provided by Warren S. Pear (UPenn) [41].

\section{Cell viability assay}

Cell viability was assessed by MTS assay (Promega). Lymphoma cell lines from Lck-Dlx 5 mice were seeded at $1 \times 10^{4}$ cells/well in 96-well plates and treated with inhibitors for 24 or $48 \mathrm{~h}$. OD value at $490 \mathrm{~nm}$ was measured 2-4 h after incubation of cells with $10 \%$ MTS using a 96-well microplate reader (BioRad).

\section{Western blot analysis}

Protein from lymphoma cell lines from $L c k-D l x 5$ mice was extracted with cell lysis buffer supplemented with $2 \mathrm{mM}$ PMSF (Cell Signaling); $50 \mu \mathrm{g} / \mathrm{well}$ proteins were loaded into Tris-Glycine SDS-PAGE gels (Invitrogen) and transferred onto PVDF membranes (Millipore). Protein blots were incubated with antibodies overnight at $4^{\circ} \mathrm{C}$. Signals were developed after incubation with HRP-conjugated secondary antibody.

\section{Microarray analysis}

Primary lymphoma samples from several different founder lines were subjected to mRNA microarray analysis 
using Affymetrix GeneChip Mouse Gene 2.0 ST Arrays (Affymetrix). Primary tumor mRNAs from $L c k$-Dlx 5 mice (F86-801, F47-918, F63-0) and Lck-MyrAkt2 (F72-3148, $3153,3237)$ mice, as well as mRNA from thymic T cells of wild-type (WT) mice, were extracted and subjected to microarray hybridization. CEL files were analyzed with Nexus expression 3.0 software (BioDiscovery, El Segundo, CA) to discover the differentially expressed genes and associated pathways. Data set has been submitted to GEO (\# GSE83685).

\section{ChIP-seq analysis}

ChIP-seq was performed and analyzed on Lck-Dlx 5 cell line F85-786 by Active Motif. Myc-Tag antibody, as well as IgG control, were used to immunoprecipitate Dlx $5 /$ chromatin complexes, and captured DNA fragments were analyzed by Illumina GA II to obtain whole-genome datasets (available at GEO \#GSE83778). MACS p-Value was used to make all peak callings.

\section{Dlx5 knockdown by retroviral-mediated short- hairpin RNA (shRNA) interference}

Sequences used for Dlx5 knockdown were selected using shRNA Design Guidelines (Ambion Technical Bulletin \#506, Life Technologies). shRNAs were synthesized, annealed and inserted into pSilencer (Invitrogen). Two constructs with best knockdown efficiency were used for experiments. Viruses were produced as described [42]. In brief, 293T cells were cotransfected with retroviral vector EcoPac and plasmid. Mouse lymphoma cell lines were infected at a MOI of 1.5 for $6 \mathrm{~h}$.

\section{Promoter and enhancer cloning}

Promoter regions and enhancer elements of mouse Notch1 and Notch 3 genes were cloned by PCR using Q5 DNA polymerase (New England Biolabs). Promoter fragments were cloned into pGL3 basic vector (Promega), and enhancer elements were inserted in the SalI site of promoter vector pGL3-core. Plasmids were transfected into 293T cells, and luciferase activity was measured using a Dual-Luciferase reporter assay kit (Promega) on an Enspire alpha plate reader (Perkin Elmer).

\section{Chromatin immunoprecipitation (ChIP) assay}

ChIP assays were performed using EZ-ChIP (Millipore) per manufacturer's instructions. Lymphoma cell lines from Lck-Dlx5 mice were fixed using 1\% formaldehyde, stopped by adding glycine to a final concentration of $125 \mathrm{mM}$. DNA:protein complexes were then resuspended in $0.1 \%$ SDS lysis buffer and sonicated into 200-1000-bp fragments. Chromatin was immunoprecipitated overnight at $4^{\circ} \mathrm{C}$ with Myc-tag antibody (Abcam). Immunocomplexes were eluted with $1 \% \mathrm{SDS}, 0.1 \mathrm{M} \mathrm{NaHCO}_{3}$, de-crosslinked with $5 \mathrm{M} \mathrm{NaCl}$, and incubated at $65^{\circ} \mathrm{C}$ overnight. PCRs of the target loci were performed using specific primers and Power SYBR Green Master Mix (Thermo Fisher Scientific).

\section{Chromosome conformation capture (CCC)}

CCC methodology and primers were as described [43]. Lymphoma cell lines from Lck-Dlx 5 mice were fixed with $2 \%$ formaldehyde for $5 \mathrm{~min}$, neutralized with glycine, and lysed with buffer containing $0.2 \% \mathrm{NP}-40,10$ $\mathrm{nM}$ Tris $\mathrm{HCl}(\mathrm{pH} 8.0)$, and $10 \mathrm{mM} \mathrm{NaCl}$ for $12 \mathrm{~h}$. DNA was digested with EcoRI or BamHI overnight, followed by ligation with T4 DNA ligase and extraction of DNA with phenol. Interaction frequencies between promoter and enhancer were analyzed by real-time PCR.

\section{Flow cytometry}

Flow cytometry was performed with a LSRII machine (Becton Dickinson) to analyze T-cell developmental and cell death markers from healthy LckDlx5 mice. CD4-APC/Cy7, CD8-PE, CD44-APC/Cy7, CD25-PE, Notch1-Alexa647 and Notch3-APC antibodies were obtained from BioLegend. Annexin V-FITC and ethidium homodimer III were from Biotium. Data were analyzed with FlowJo software.

\section{ACKNOWLEDGMENTS AND FUNDING}

The authors thank the Transgenic Mouse, Genomics, Flow Cytometry, Laboratory Animal, and DNA Sequencing Facilities at Fox Chase Cancer Center for assistance.

\section{CONFLICTS OF INTEREST}

None.

\section{GRANT SUPPORT}

This work was supported by NCI grants CA77429 and CA06927, and an appropriation from the Commonwealth of Pennsylvania.

\section{REFERENCES}

1. Homminga I, Pieters R, Meijerink JP. NKL homeobox genes in leukemia. Leukemia. 2012; 26:572-581.

2. Hatano $\mathrm{M}$, Roberts $\mathrm{CW}$, Minden $\mathrm{M}$, Crist WM, Korsmeyer SJ. Deregulation of a homeobox gene, HOX11, by the $\mathrm{t}(10 ; 14)$ in T cell leukemia. Science. 1991; 253:79-82.

3. Soulier J, Clappier E, Cayuela JM, Regnault A, GarciaPeydro M, Dombret H, Baruchel A, Toribio ML, Sigaux F. 
HOXA genes are included in genetic and biologic networks defining human acute T-cell leukemia (T-ALL). Blood. 2005; 106:274-286.

4. Panganiban G, Rubenstein JL. Developmental functions of the Distal-less/Dlx homeobox genes. Development. 2002; 129:4371-4386.

5. Depew MJ, Liu JK, Long JE, Presley R, Meneses JJ, Pedersen RA, Rubenstein JL. Dlx5 regulates regional development of the branchial arches and sensory capsules. Development. 1999; 126:3831-3846.

6. Tan Y, Timakhov RA, Rao M, Altomare DA, Xu J, Liu Z, Gao Q, Jhanwar SC, Di Cristofano A, Wiest DL, Knepper JE, Testa JR. A novel recurrent chromosomal inversion implicates the homeobox gene Dlx5 in T-cell lymphomas from Lck-Akt2 transgenic mice. Cancer Res. 2008; 68:1296-1302.

7. Xu J, Testa JR. DLX5 (distal-less homeobox 5) promotes tumor cell proliferation by transcriptionally regulating MYC. J Biol Chem. 2009; 284:20593-20601.

8. Kato T, Sato N, Takano A, Miyamoto M, Nishimura H, Tsuchiya E, Kondo S, Nakamura Y, Daigo Y. Activation of placenta-specific transcription factor distal-less homeobox 5 predicts clinical outcome in primary lung cancer patients. Clin Cancer Res. 2008; 14:2363-2370.

9. Morini M, Astigiano S, Gitton Y, Emionite L, Mirisola V, Levi G, Barbieri O. Mutually exclusive expression of DLX2 and DLX5/6 is associated with the metastatic potential of the human breast cancer cell line MDA-MB-231. BMC cancer. 2010; 10:649.

10. Yan ZH, Bao ZS, Yan W, Liu YW, Zhang CB, Wang HJ, Feng Y, Wang YZ, Zhang W, You G, Zhang QG, Jiang T. Upregulation of DLX2 confers a poor prognosis in glioblastoma patients by inducing a proliferative phenotype. Curr Mol Med. 13:438-445.

11. Tan Y, Cheung M, Pei J, Menges CW, Godwin AK, Testa JR. Upregulation of DLX5 promotes ovarian cancer cell proliferation by enhancing IRS-2-AKT signaling. Cancer Res. 2010; 70:9197-9206.

12. Woodside KJ, Shen H, Muntzel C, Daller JA, Sommers CL, Love PE. Expression of Dlx and Lhx family homeobox genes in fetal thymus and thymocytes. Gene Expr Patterns. 2004; 4:315-320.

13. Tan Y, Sementino E, Pei J, Kadariya Y, Ito TK, Testa JR. Cotargeting of Akt and Myc inhibits viability of lymphoma cells from Lck-Dlx5 mice. Cancer Biol Ther. 2015; 16:580-588.

14. Grabher C, von Boehmer H, Look AT. Notch 1 activation in the molecular pathogenesis of T-cell acute lymphoblastic leukaemia. Nat Rev Cancer. 2006; 6:347-359.

15. Jonveaux P, Berger R. Infrequent mutations in the P53 gene in primary human T-cell acute lymphoblastic leukemia. Leukemia. 1991; 5:839-840.

16. Petit B, Leroy K, Kanavaros P, Boulland ML, DruetCabanac M, Haioun C, Bordessoule D, Gaulard P. Expression of p53 protein in $\mathrm{T}$ - and natural killer-cell lymphomas is associated with some clinicopathologic entities but rarely related to p53 mutations. Hum Pathol. 2001; 32:196-204.

17. Raetz EA, Perkins SL, Bhojwani D, Smock K, Philip M, Carroll WL, Min DJ. Gene expression profiling reveals intrinsic differences between T-cell acute lymphoblastic leukemia and T-cell lymphoblastic lymphoma. Pediatr Blood Cancer. 2006; 47:130-140.

18. Ross ME, Zhou X, Song G, Shurtleff SA, Girtman K, Williams WK, Liu HC, Mahfouz R, Raimondi SC, Lenny N, Patel A, Downing JR. Classification of pediatric acute lymphoblastic leukemia by gene expression profiling. Blood. 2003; 102:2951-2959.

19. Iqbal J, Weisenburger DD, Greiner TC, Vose JM, McKeithan T, Kucuk C, Geng H, Deffenbacher K, Smith L, Dybkaer K, Nakamura S, Seto M, Delabie J, et al. Molecular signatures to improve diagnosis in peripheral T-cell lymphoma and prognostication in angioimmunoblastic T-cell lymphoma. Blood. 2010; 115:1026-1036.

20. Medyouf H, Gao X, Armstrong F, Gusscott S, Liu Q, Gedman AL, Matherly LH, Schultz KR, Pflumio F, You MJ, Weng AP. Acute T-cell leukemias remain dependent on Notch signaling despite PTEN and INK4A/ARF loss. Blood. 2010; 115:1175-1184.

21. King B, Trimarchi T, Reavie L, Xu L, Mullenders J, Ntziachristos P, Aranda-Orgilles B, Perez-Garcia A, Shi J, Vakoc C, Sandy P, Shen SS, Ferrando A, et al. The ubiquitin ligase FBXW7 modulates leukemia-initiating cell activity by regulating MYC stability. Cell. 2013; 153:1552-1566.

22. Speleman F, Cauwelier B, Dastugue N, Cools J, Verhasselt B, Poppe B, Van Roy N, Vandesompele J, Graux C, Uyttebroeck A, Boogaerts M, De Moerloose B, Benoit $Y$, et al. A new recurrent inversion, inv(p15q34), leads to transcriptional activation of HOXA10 and HOXA11 in a subset of T-cell acute lymphoblastic leukemias. Leukemia. 2005; 19:358-366.

23. Ferrell CM, Dorsam ST, Ohta H, Humphries RK, Derynck MK, Haqq C, Largman C, Lawrence HJ. Activation of stem-cell specific genes by HOXA9 and HOXA10 homeodomain proteins in CD34+ human cord blood cells. Stem Cells. 2005; 23:644-655.

24. Radtke F, Wilson A, Stark G, Bauer M, van Meerwijk J, MacDonald HR, Aguet M. Deficient T cell fate specification in mice with an induced inactivation of Notch1. Immunity. 1999; 10:547-558.

25. Pui JC, Allman D, Xu L, DeRocco S, Karnell FG, Bakkour S, Lee JY, Kadesch T, Hardy RR, Aster JC, Pear WS. Notch1 expression in early lymphopoiesis influences B versus T lineage determination. Immunity. 1999; 11:299-308.

26. Ciofani M, Zuniga-Pflucker JC. Notch promotes survival of pre-T cells at the beta-selection checkpoint by regulating cellular metabolism. Nat Immunol. 2005; 6:881-888.

27. Aifantis I, Raetz E, Buonamici S. Molecular pathogenesis of T-cell leukaemia and lymphoma. Nat Rev Immunol. 2008; 8:380-390. 
28. Pear WS, Aster JC, Scott ML, Hasserjian RP, Soffer B, Sklar J, Baltimore D. Exclusive development of T cell neoplasms in mice transplanted with bone marrow expressing activated Notch alleles. J Exp Med. 1996; 183:2283-2291.

29. Chiang MY, Xu L, Shestova O, Histen G, L'Heureux S, Romany C, Childs ME, Gimotty PA, Aster JC, Pear WS. Leukemia-associated NOTCH1 alleles are weak tumor initiators but accelerate K-ras-initiated leukemia. J Clin Invest. 2008; 118:3181-3194.

30. Bellavia D, Campese AF, Checquolo S, Balestri A, Biondi A, Cazzaniga G, Lendahl U, Fehling HJ, Hayday AC, Frati L, von Boehmer H, Gulino A, Screpanti I. Combined expression of pTalpha and Notch3 in $T$ cell leukemia identifies the requirement of preTCR for leukemogenesis. Proc Natl Acad Sci USA. 2002; 99:3788-3793.

31. Brooks YS, Ostano P, Jo SH, Dai J, Getsios S, Dziunycz P, Hofbauer GF, Cerveny K, Chiorino G, Lefort K, Dotto GP. Multifactorial ERbeta and NOTCH1 control of squamous differentiation and cancer. J Clin Invest. 2014; 124:2260-2276.

32. Durinck K, Van Loocke W, Van der Meulen J, Van de Walle I, Ongenaert M, Rondou P, Wallaert A, de Bock CE, Van Roy N, Poppe B, Cools J, Soulier J, Taghon T, et al. Characterization of the genome-wide TLX1 binding profile in T-cell acute lymphoblastic leukemia. Leukemia. 2015; 29:2317-2327.

33. Sharfe N, Roifman CM. Differential association of phosphatidylinositol 3-kinase with insulin receptor substrate (IRS)-1 and IRS-2 in human thymocytes in response to IL-7. J Immunol. 1997; 159:1107-1114.

34. Wurster AL, Withers DJ, Uchida T, White MF, Grusby MJ. Stat6 and IRS-2 cooperate in interleukin 4 (IL-4)-induced proliferation and differentiation but are dispensable for IL4-dependent rescue from apoptosis. Mol Cell Biol. 2002; 22:117-126.

35. Dibirdik I, Langlie MC, Ledbetter JA, Tuel-Ahlgren L, Obuz V, Waddick KG, Gajl-Peczalska K, Schieven GL, Uckun FM. Engagement of interleukin-7 receptor stimulates tyrosine phosphorylation, phosphoinositide turnover, and clonal proliferation of human T-lineage acute lymphoblastic leukemia cells. Blood. 1991; 78:564-570.

36. Zenatti PP, Ribeiro D, Li W, Zuurbier L, Silva MC, Paganin M, Tritapoe J, Hixon JA, Silveira AB, Cardoso BA, Sarmento LM, Correia N, Toribio ML, et al. Oncogenic
IL7R gain-of-function mutations in childhood T-cell acute lymphoblastic leukemia. Nat Genet. 2011; 43:932-939.

37. Gutierrez A, Sanda T, Grebliunaite R, Carracedo A, Salmena L, Ahn Y, Dahlberg S, Neuberg D, Moreau LA, Winter SS, Larson R, Zhang J, Protopopov A, et al. High frequency of PTEN, PI3K, and AKT abnormalities in T-cell acute lymphoblastic leukemia. Blood. 2009; 114:647-650.

38. Jotta PY, Ganazza MA, Silva A, Viana MB, da Silva MJ, Zambaldi LJ, Barata JT, Brandalise SR, Yunes JA. Negative prognostic impact of PTEN mutation in pediatric T-cell acute lymphoblastic leukemia. Leukemia. 2010; 24:239-242.

39. Dearth RK, Cui X, Kim HJ, Kuiatse I, Lawrence NA, Zhang X, Divisova J, Britton OL, Mohsin S, Allred DC, Hadsell DL, Lee AV. Mammary tumorigenesis and metastasis caused by overexpression of insulin receptor substrate 1 (IRS-1) or IRS-2. Mol Cell Biol. 2006; 26:9302-9314.

40. Timakhov RA, Tan Y, Rao M, Liu Z, Altomare DA, Pei J, Wiest DL, Favorova OO, Knepper JE, Testa JR. Recurrent chromosomal rearrangements implicate oncogenes contributing to T-cell lymphomagenesis in Lck-MyrAkt2 transgenic mice. Genes Chromosomes Cancer. 2009; 48:786-794.

41. Maillard I, Tu L, Sambandam A, Yashiro-Ohtani Y, Millholland J, Keeshan K, Shestova O, Xu L, Bhandoola A, Pear WS. The requirement for Notch signaling at the betaselection checkpoint in vivo is absolute and independent of the pre-T cell receptor. J Exp Med. 2006; 203:2239-2245.

42. Tan Y, Dourdin N, Wu C, De Veyra T, Elce JS, Greer PA. Ubiquitous calpains promote caspase-12 and JNK activation during endoplasmic reticulum stress-induced apoptosis. J Biol Chem. 2006; 281:16016-16024.

43. Hagege H, Klous P, Braem C, Splinter E, Dekker J, Cathala G, de Laat W, Forne T. Quantitative analysis of chromosome conformation capture assays (3C-qPCR). Nat Protoc. 2007; 2:1722-1733.

44. Altomare DA, Zhang LL, Deng J, Di Cristofano A, KleinSzanto AJ, Kumar R, Testa JR. GSK690693 Delays Tumor Onset and Progression in Genetically Defined Mouse Models Expressing Activated Akt. Clinical Cancer Research. 2010; 16:486-496. 